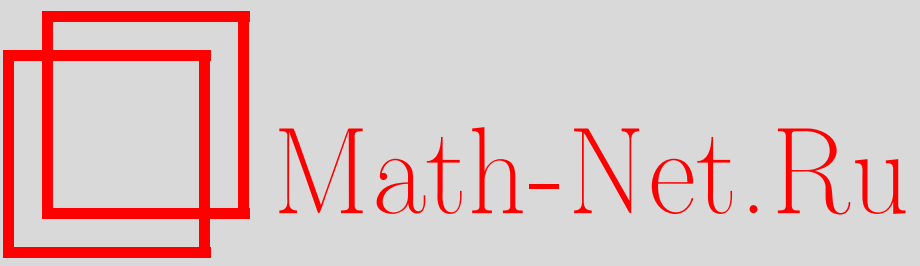

А. Г. Баскаков, Г. В. Гаркавенко, Н. Б. Ускова, О матрицах с суммируемыми диагоналями, Итоги науки и техн. Сер. Соврем. мат. и ее прил. Темат. обз., 2021, том 194, 23-37

DOI: https://doi.org/10.36535/0233-6723-2021-194-23-37

Использование Общероссийского математического портала Math-Net.Ru подразумевает, что вы прочитали и согласны с пользовательским соглашением

http://www . mathnet.ru/rus/agreement

Параметры загрузки:

IP: 52.87 .193 .239

26 апреля 2023 г., 02:54:34 


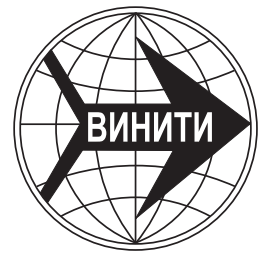

ИТОГИ НАУКИ И ТЕХНИКИ.

Современная математика и ее приложения.

Тематические обзоры.

Том 194 (2021). C. 23-37

DOI: 10.36535/0233-6723-2021-194-23-37

УДК 517.927

\title{
О МАТРИЦАХ С СУММИРУЕМЫМИ ДИАГОНАЛЯМИ
}

\author{
(c) 2021 г. A. Г. БАСКАКОВ, Г. В. ГАРКАВЕНКО, Н. Б. УСКОВА
}

\begin{abstract}
АннотАция. В работе рассматриваются основные понятия и факты, связанные с бесконечными матрицами. Матричный (операторный) подход применен к доказательству теоремы Винера об абсолютно сходящихся рядах Фурье. Изучается модификация метода подобных операторов, связанная с уравнением Фридрихса. С помощью метода подобных операторов осуществляется приведение строго нижнетреугольной матрицы с суммируемыми диагоналями к диагональному (блочно-диагональному) виду, что позволяет найти ее спектр.
\end{abstract}

Ключевые слова: матрица с суммируемыми диагоналями, метод подобных операторов, спектр.

\section{ON MATRICES WITH SUMMABLE DIAGONALS}

\author{
(c) 2021 A. G. BASKAKOV, G. V. GARKAVENKO, N. B. USKOVA
}

\begin{abstract}
In this paper, basic concepts and facts related to infinite matrices are considered. The matrix (operator) approach is applied to the proof of Wiener's theorem on absolutely convergent Fourier series. A modification of the method of similar operators related to the Friedrichs equation is studied. Using the method of similar operators, we reduce a strictly lower triangular matrix with summable diagonals to the diagonal (block diagonal) form; this allows one to find the spectrum.
\end{abstract}

Keywords and phrases: matrix with summable diagonals, method of similar operators, spectrum.

AMS Subject Classification: $47 \mathrm{~B} 38$

1. Введение. Пусть $\mathbb{Z}$ - кольцо целых чисел, $\mathbb{N}$ - множество натуральных чисел, $\mathbb{Z}_{+}=\mathbb{N} \cup\{0\}$. Всюду далее через $\mathbb{J}$ и $\mathbb{J}^{\prime}$ обозначено любое из множеств $\mathbb{N}, \mathbb{Z}, \mathbb{Z}+$ или их конечные подмножества вида $\left\{m \in \mathbb{N}, m \leqslant N_{0}\right\},\left\{m \in \mathbb{Z}_{+}, m \leqslant N_{0}\right\},\left\{m \in \mathbb{Z},|m| \leqslant N_{0}\right\},\left\{m \in \mathbb{Z},|m| \leqslant \mathbb{N}_{0}, m \leqslant 0\right\}$, где $N_{0} \in \mathbb{N}$.

Определение 1. Отображение $\mathcal{A}: \mathbb{J} \times \mathbb{J}^{\prime} \rightarrow \mathbb{C}$ называется матрицей с комплексными элементами. Элементы матрицы будем обозначать через $\mathcal{A}(n, m), n \in \mathbb{J}, m \in \mathbb{J}^{\prime}$.

Отметим, что используются и другие определения матрицы (см. например, $[8,15])$ (даже в случае бесконечных матриц). Но в некоторых прикладных вопросах, например, в теории игр, также удобно представление матрицы как отображения из декартова произведения некоторого подмножества индексов во множество рациональных чисел (см. [12]).

Определение 2. Если множества $\mathbb{J}$ и $\mathbb{J}^{\prime}$ совпадают, то матрицу будем называть квадратной. Если же оба множества $\mathbb{J}$ и $\mathbb{J}^{\prime}$ конечны, то матрицу будем называть конечной матрицей размерности $n \times m$, где $n$ - количество элементов в $\mathbb{J}$ и $m$ - количество элементов в $\mathbb{J}^{\prime}$.

Далее нас будут интересовать бесконечные матрицы. Множество матриц образует линейное пространство, обозначаемое далее символом $\operatorname{Matr}\left(\mathbb{J} \times \mathbb{J}^{\prime}, \mathbb{C}\right)$.

Работа выполнена при поддержке Российского фонда фундаментальных исследований (проект № 19-01-00732а). 
В работе рассматриваются вопросы диагонализации (блочной диагонализации) специальных классов бесконечных матриц, а именно, бесконечных матриц с суммируемыми диагоналями (см. определение 15) с помощью преобразования подобия. Отметим работы [9,20], в которых рассматривался вопрос диагонализации некоторых классов линейных операторов. Методом приведения матриц к диагональному (блочно-диагональному) виду служит метод подобных операторов (см. $[1-4,9])$. Однако при рассмотрении строго нижнетреугольных матриц с суммируемыми диагоналями (см. определение 15) используется отличная от традиционной модификация метода подобных операторов, использующая уравнение Фридрихса (см. [2]). Это позволяет доказать теорему о подобии возмущенной матрицы и невозмущенной диагональной матрицы. Ранее уравнение Фридрихса применялось только в [17]. Поэтому сначала разрабатывается соответствующая модификация метода подобных операторов, выводятся условия ее применимости, доказываются условия разрешимости соответствующих операторных уравнений метода подобных операторов. Потом все это применяется к исследуемому оператору. Отметим работы $[10,11]$, в которых исследовались спектральные свойства разностных операторов с растущим потенциалом, заданных своими трехдиагональными матрицами. Там тоже не применялась модификация метода подобных операторов, связанная с уравнением Фридрихса.

Статья построена следующим образом. Вначале вводятся основные понятия и определения, используемые далее в работе. При этом также получается доказательство классической теоремы Винера с использованием рядов Фурье операторов с суммируемыми диагоналями. Затем излагается с доказательством используемая далее модификация метода подобных операторов, опирающаяся на уравнение Фридрихса. И, наконец, стандартная схема метода подобных операторов применяется для приведения к диагональному или блочно-диагональному виду изучаемой строго нижнетреугольной матрицы с суммируемыми диагоналями, что позволяет найти ее спектр.

\section{2. Основные определения, леммы и теоремы.}

2.1. Основные понятия. Введем специальные классы матриц, как и в случае матриц конечной размерности.

Определение 3. Матрица $\mathcal{A} \in \operatorname{Matr}\left(\mathbb{J} \times \mathbb{J}^{\prime}, \mathbb{C}\right)$ называется верхнетреугольной, если $\mathcal{A}(i, j)=0$ для всех $i>j, i \in \mathbb{J}, j \in \mathbb{J}^{\prime}$. Матрица $\mathcal{A} \in \operatorname{Matr}\left(\mathbb{J} \times \mathbb{J}^{\prime}, \mathbb{C}\right)$ называется нижнетреугольной, если $\mathcal{A}(i, j)=0$ для всех $i<j, i \in \mathbb{J}, j \in \mathbb{J}^{\prime}$. Матрица $\mathcal{A} \in \operatorname{Matr}\left(\mathbb{J} \times \mathbb{J}^{\prime}, \mathbb{C}\right)$ называется диагональной, если $\mathcal{A}(i, j)=0$ при $i \neq j, i, j \in \mathbb{J}\left(\right.$ т.е. $\left.\mathbb{J}=\mathbb{J}^{\prime}\right)$.

Матрица $\mathcal{A} \in \operatorname{Matr}\left(\mathbb{J} \times \mathbb{J}^{\prime}, \mathbb{C}\right)$ называется строго верхнетреугольной, если $\mathcal{A}(i, j)=0$ для всех $i \geqslant j, i \in \mathbb{J}, j \in \mathbb{J}^{\prime}$. Матрица $\mathcal{A} \in \operatorname{Matr}\left(\mathbb{J} \times \mathbb{J}^{\prime}, \mathbb{C}\right)$ называется строго нижнетреугольной, если $\mathcal{A}(i, j)=0$ для всех $i \leqslant j, i \in \mathbb{J}, j \in \mathbb{J}^{\prime}$.

Определение 4. Матрица $\mathcal{A}^{T} \in \operatorname{Matr}\left(\mathbb{J}^{\prime} \times \mathbb{J}, \mathbb{C}\right)$ называется транспонированной для матрицы $\mathcal{A} \in \operatorname{Matr}\left(\mathbb{J} \times \mathbb{J}^{\prime}, \mathbb{C}\right)$, если $\mathcal{A}^{T}(i, j)=\mathcal{A}(j, i), j \in \mathbb{J}, i \in \mathbb{J}^{\prime}$.

Определение 5. Подматрицей $\mathcal{A}_{0} \in \operatorname{Matr}\left(\mathbb{J}_{0} \times \mathbb{J}_{0}^{\prime}, \mathbb{C}\right)$ матрицы $\mathcal{A} \in \operatorname{Matr}\left(\mathbb{J} \times \mathbb{J}^{\prime}, \mathbb{C}\right)$ называется сужение матрицы $\mathcal{A}$ на декартово произведение двух конечных множеств $\mathbb{J}_{0}$ и $\mathbb{J}_{0}^{\prime}$.

Минором $k$-го порядка матрицы $\mathcal{A} \in \operatorname{Matr}\left(\mathbb{J} \times \mathbb{J}^{\prime}, \mathbb{C}\right)$ называется квадратная подматрица $M_{\mathcal{A}}: \mathbb{J}_{k} \times \mathbb{J}_{k} \rightarrow \mathbb{C}$, где множество $\mathbb{J}_{k}$ состоит из $k$ элементов.

Как и в случае конечных матриц, рангом матрицы называется наиболыший порядок ее отличного от нуля минора. Заметим, что в рассматриваемом случае ранг матрицы может быть равен бесконечности.

Пример 1. Матрица $\mathcal{J} \in \operatorname{Matr}\left(\mathbb{N}^{2}, \mathbb{C}\right)$, где $\mathcal{J}(i, j)=1$ для всех $i, j \in \mathbb{J}$ имеет ранг 1 , т.е. $\operatorname{Rg} \mathcal{J}=1$.

Определение 6. Последовательность $c_{k}: \mathbb{J} \rightarrow \mathbb{C}$, элементы которой определяются формулой $c_{k}(n)=\mathcal{A}(n, k), n \in \mathbb{J}, k \in \mathbb{J}^{\prime}$ называется $k$-ым столбцом матрицы $\mathcal{A}$. Последовательность $b_{k}: \mathbb{J}^{\prime} \rightarrow \mathbb{C}$, где $b_{k}(n)=\mathcal{A}(k, n), n \in \mathbb{J}^{\prime}, k \in \mathbb{J}$ называется $k$-й строкой матрицы $\mathcal{A}$. Последовательность $a_{k}: \mathbb{Z} \rightarrow \mathbb{C}$, где $a_{k}(n)=\mathcal{A}(n, m), n-m=k, n \in \mathbb{J}, m \in \mathbb{J}^{\prime}, k \in \mathbb{Z}$ называется $k$-й диагональю матрицы $\mathcal{A}$. При $k=0$ диагональ называется главной. 
Введем в рассмотрение банахово пространство $l_{p}(\mathbb{J}), 1 \leqslant p<\infty$ последовательностей комплексных чисел $x: \mathbb{J} \rightarrow \mathbb{C}$, суммируемых со степенью $1 \leqslant p<\infty$ с нормой

$$
\|x\|_{p}=\left(\sum_{n \in \mathbb{J}}|x(n)|^{p}\right)^{1 / p} .
$$

При $p=\infty$ норма задается формулой

$$
\|x\|_{\infty}=\sup _{n \in \mathbb{J}}|x(n)|, \quad x: \mathbb{J} \rightarrow \mathbb{C}, \quad x \in l_{\infty} .
$$

Отметим, что при $p=2$ пространство $l_{2}(\mathbb{J})$ является гильбертовым, со скалярным произведением

$$
(x, y)=\sum_{n \in \mathbb{J}} x(n) \overline{y(n)}, \quad x, y: \mathbb{J} \rightarrow \mathbb{C}, \quad x, y \in l_{2}(\mathbb{J}) .
$$

В каждом из пространств $l_{p}(\mathbb{J}), 1 \leqslant p<\infty$ последовательности $e_{k}, k \in \mathbb{J}$, такие, что $e_{k}(n)=1$ при $n=k$ и $e_{k}(n)=0$ при $n \neq k, k, n \in \mathbb{J}$, образуют безусловный базис. В пространстве $l_{2}(\mathbb{J})$ введенный базис является ортонормированным.

Определение 7. Последовательность $x \in l_{p}(\mathbb{J}), 1 \leqslant p<\infty$, называется финитной, если множество индексов $n \in \mathbb{J}$, для которых $x(n) \neq 0$, конечно.

Через $l_{p, 0}$ обозначим подпространство финитных последовательностей из $l_{p}(\mathbb{J})$. Финитные последовательности плотны в $l_{p}(\mathbb{J})$, при $p \neq \infty, \bar{l}_{p, 0}=l_{p}(\mathbb{J})$.

Пусть $\mathcal{X}$ - абстрактное банахово пространство. Символом End $\mathcal{X}$ обозначена банахова алгебра всех ограниченных линейных операторов, действующих в банаховом пространстве $\mathcal{X}$ с нормой

$$
\|X\|=\sup _{\|x\| \leqslant 1}\|X x\|, \quad X \in \operatorname{End} \mathcal{X}, \quad x \in \mathcal{X} .
$$

Далее будем рассматривать бесконечные матрицы, для которых $\mathbb{J}=\mathbb{J}^{\prime}$. Каждой матрице $\mathcal{A} \in \operatorname{Matr}\left(\mathbb{J}^{2}, \mathbb{C}\right)$ сопоставим линейный оператор $A: l_{p, 0} \rightarrow l_{p, 0}, 1 \leqslant p \leqslant \infty$, положив

$$
(A x)(k)=\sum_{j \in \mathbb{J}} \mathcal{A}(k, j) x(j), \quad x \in l_{p, 0}, \quad k \in \mathbb{J} .
$$

Определение 8. Матрицу $\mathcal{A} \in \operatorname{Matr}\left(\mathbb{J}^{2}, \mathbb{C}\right)$ назовем $p$-правильной для некоторого $1 \leqslant p \leqslant \infty$, если соответствующий оператор $A$ допускает замкнутое расширение на пространство $l_{p}(\mathbb{J})$ до некоторого оператора. Обозначим это расширение тем же символом $A: D(A) \subset l_{p}(\mathbb{J}) \rightarrow l_{p}(\mathbb{J})$, $1 \leqslant p \leqslant \infty$.

Если матрица является $p$-правильной для любого $1 \leqslant p \leqslant \infty$, то будем просто называть ее правильной.

Пример 2. Матрица $\mathcal{J}$ из примера 1 не является $\infty$-правильной. Покажем это.

$\mathrm{B}$ пространстве $l_{\infty}$ зададим семейство последовательностей $x_{n}: l_{\infty} \rightarrow \mathbb{C}, n \geqslant 1$, формулой

$$
x_{n}=(\underbrace{\frac{1}{n}, \frac{1}{n}, \frac{1}{n}, \ldots, \frac{1}{n}}_{n}, 0,0, \ldots) .
$$

Тогда

$$
\left(A x_{n}\right)(k)=\sum_{i=1}^{n} \frac{1}{n}=1
$$

для всех $k \in \mathbb{N}$. Но последовательность последовательностей $\left(x_{n}, n \geqslant 1\right)$ сходится к нулевой последовательности, а $A 0=0$.

Определение 9. Правильная матрица $\mathcal{A} \in \operatorname{Matr}\left(\mathbb{J}^{2}, \mathbb{C}\right)$ называется обратимой, если обратим соответствующий линейный оператор $A: D(A) \subset l_{p}(\mathbb{J}) \rightarrow l_{p}(\mathbb{J})$. 
Определение 10. Матрица $\mathcal{A} \in \operatorname{Matr}\left(\mathbb{J}^{2}, \mathbb{C}\right)$ называется матрицей с равномерно ограниченными элементами, если

$$
\sup _{i, j \in \mathbb{J}}|A(i, j)|=c_{\mathcal{A}}<\infty .
$$

Определение 11. Матрицу $\mathcal{A} \in \operatorname{Matr}\left(\mathbb{J}^{2}, \mathbb{C}\right)$ назовем ограниченной, если соответствующий оператор $A: l_{p}(\mathbb{J}) \rightarrow l_{p}(\mathbb{J})$ ограничен для некоторого $1 \leqslant p \leqslant \infty$.

Имеют место следующие очевидные утверждения.

Лемма 1. Каждая ограниченная матрииа является р-правильной.

Лемма 2. Каждая диагональная матрица с $\inf _{i \in \mathbb{J}}|\mathcal{A}(i, i)|>0$ является обратимой правильной матрищей.

Пусть $\mathcal{A} \in \operatorname{Matr}\left(\mathbb{J}^{2}, \mathbb{C}\right)$ - диагональная матрица. Тогда соответствующий линейный оператор $A: D(A) \subset l_{p}(\mathbb{J}) \rightarrow l_{p}(\mathbb{J})$ действует по формуле $(A x)(k)=\mathcal{A}(k, k) x(k), k \in \mathbb{J}$, и имеет область определения

$$
D(A)=\left\{x \in l_{p}(\mathbb{J}), \sum_{k \in \mathbb{J}}|A(k, k) x(k)|^{p}<\infty\right\} \text { для } 1 \leqslant p<\infty
$$

или

$$
D(A)=\left\{x \in l_{\infty}, \sup _{k \in \mathbb{J}}|A(k, k) x(k)|<\infty\right\} .
$$

Лемма 3. Область определения $D(A)$ оператора $A$ плотна в $l_{p}(\mathbb{J}), 1 \leqslant p<\infty$.

Утверждение леммы 3 следует из плотности финитных последовательностей в $l_{p}(\mathbb{J})$.

Напомним некоторые определения из теории операторов (см. $[2,16])$.

Определение 12. Графиком линейного оператора $A: D(A) \subset \mathcal{X} \rightarrow \mathcal{X}$ называется подмножество векторов из $\mathcal{X} \times \mathcal{X}$ вида $(x, A x), x \in D(A)$.

Определение 13. Оператор $A: D(A) \subset \mathcal{X} \rightarrow \mathcal{X}$ называется замкнутым, если его график является замкнутым подмножеством в $\mathcal{X} \times \mathcal{X}$.

В качестве нормы в $\mathcal{X} \times \mathcal{X}$ можно, например, взять норму графика $\|(x, y)\|=\|x\|+\|y\|$. Понятие замкнутости для оператора $A: D(A) \subset \mathcal{X} \rightarrow \mathcal{X}$ означает, что из условий

$$
x_{n} \in D(A), \quad \lim _{n \rightarrow \infty} x_{n}=x_{0}, \quad \lim _{n \rightarrow \infty} A x_{n}=y_{0}
$$

следует, что $x_{0} \in D(A)$ и $A x_{0}=y_{0}$. Поэтому понятие правильной матрицы очень тесно связано с понятием замкнутости соответствующего оператора.

Теперь сделаем обратный шаг. Пусть $A: D(A) \subset l_{p}(\mathbb{J}) \rightarrow l_{p}(\mathbb{J}), 1 \leqslant p<\infty$-замкнутый оператор. Определим его матрицу следующим образом. Пусть его область определения $D(A)$ содержит базис $e_{k}, k \in \mathbb{J}$. Тогда элементы матрицы $\mathcal{A} \in \operatorname{Matr}\left(\mathbb{J} \times \mathbb{J}^{\prime}, \mathbb{C}\right)$ оператора $A$ определяются формулой

$$
\mathcal{A}(i, j)=\left(A e_{j}\right)(i), \quad i \in \mathbb{J}, \quad j \in \mathbb{J}^{\prime} .
$$

Замечание 1. Матрица замкнутого оператора всегда правильная.

Лемма 4. Матрица замкнутого оператора одинакова в любом из пространств $l_{p}(\mathbb{J})$, $1 \leqslant p \leqslant \infty$.

Если матрица определяет ограниченный оператор, то можно считать, что норма матрицы равна норме соответствующего оператора $A: l_{p}(\mathbb{J}) \rightarrow l_{p}(\mathbb{J})$. Так, например, если $A: l_{1}(\mathbb{J}) \rightarrow l_{1}(\mathbb{J})$, то

$$
\|A\|_{l_{1}}=\sup _{j \in \mathbb{J}} \sum_{i \in \mathbb{J}}|\mathcal{A}(i, j)| .
$$


Определение 14. Матрица $\mathcal{A} \in \operatorname{Matr}\left(\mathbb{J}^{2}, \mathbb{C}\right)$ называется матрицей Гильберта - Шмидта, если $\sum_{i, j \in \mathbb{J}}|A(i, j)|^{2}<\infty$.

Замечание 2. Не всякая матрица конечного ранга является матрицей Гильберта-Шмидта. Например, матрица из примера 1 не является таковой.

Пусть $\mathcal{A} \in \operatorname{Matr}\left(\mathbb{J}^{2}, \mathbb{C}\right)$ - правильная матрица. Так как соответствующий оператор $A$ в этом случае имеет плотную в $l_{2}(\mathbb{J})$ область определения $D(A)$, то по определению сопряженный к нему оператор $A^{*}: D\left(A^{*}\right) \subset l_{2}(\mathbb{J}) \rightarrow l_{2}(\mathbb{J})$ имеет матрицу $\mathcal{A}^{T}$.

Пусть $\mathcal{A}, \mathcal{B} \in \operatorname{Matr}\left(\mathbb{J}^{2}, \mathbb{C}\right)$. Определим произведение $\mathcal{A B}$ двух замкнутых матриц следующим образом. Рассмотрим расширения $A: D(A) \subset l_{p}(\mathbb{J}) \rightarrow l_{p}(\mathbb{J}), B: D(B) \subset l_{p}(\mathbb{J}) \rightarrow l_{p}(\mathbb{J})$ операторов, определяемых матрицами $\mathcal{A}, \mathcal{B}$ соответственно. Определим $\mathcal{A B}$ как матрицу оператора $C$ произведения операторов $A$ и $B$.

Следует отметить, что обычное произведение двух бесконечных матриц может не существовать.

Лемма 5. Если матрица $\mathcal{A} \in \operatorname{Matr}\left(\mathbb{J}^{2}, \mathbb{C}\right)$ является правильной, а матрица $\mathcal{B} \in \operatorname{Matr}\left(\mathbb{J}^{2}, \mathbb{C}\right)$ ограниченная, то $\mathcal{A B}$ - правильная матрица. Если же $\mathcal{B}$-обратимая матрица, то $\mathcal{B} \mathcal{A}$ - правильная матрица.

Лемма 6. Матрица $\mathcal{A} \in \operatorname{Matr}\left(\mathbb{J}^{2}, \mathbb{C}\right)$ правильная, если все ее строки суммируемы и нет нулевых строк.

Доказательство. Построим по матрице $\mathcal{A} \in \operatorname{Matr}\left(\mathbb{J}^{2}, \mathbb{C}\right)$ последовательность $f: \mathbb{J} \rightarrow \mathbb{R}_{+}$следующим образом. Положим $f(k)=\sum_{j \in \mathbb{J}}|A(k, j)|$. Введем в рассмотрение диагональную матрицу $\mathcal{F} \in \operatorname{Matr}\left(\mathbb{J}^{2}, \mathbb{C}\right)$ с элементами $\mathcal{F}(i, i)=f(i), i \in \mathbb{J}, \mathcal{F}(i, j)=0, i \neq j, i, j \in \mathbb{J}$. Очевидно, что диагональная матрица $\mathcal{F}$ правильная и обратимая. Представим матрицу $\mathcal{A}$ в виде $\mathcal{A}=\mathcal{F F}^{-1} \mathcal{A}=\mathcal{F} \mathcal{B}$, где $\mathcal{B}=\mathcal{F}^{-1} \mathcal{A}$ - ограниченная матрица и $\mathcal{F}$ - правильная. Тогда по лемме 3 матрица $\mathcal{A}$ правильная.

Введем в рассмотрение последовательность $d_{\mathcal{A}}: \mathbb{Z} \rightarrow \mathbb{R}_{+}$, положив $d_{\mathcal{A}}(k)=\sup _{i-j=k}|\mathcal{A}(i, j)|$, $i, j \in \mathbb{J}, k \in \mathbb{Z}$. Отметим, что последовательность $d_{\mathcal{A}}: \mathbb{Z} \rightarrow \mathbb{R}_{+}$определена и для прямоугольных матриц, т.е. когда $\mathbb{J} \neq \mathbb{J}^{\prime}$.

Определение 15. Матрица $\mathcal{A} \in \operatorname{Matr}\left(\mathbb{J}^{2}, \mathbb{C}\right)$ называется матрицей с суммируемыми диагоналями, если конечна величина $d(\mathcal{A})=\sum_{n \in \mathbb{Z}} d_{\mathcal{A}}(n)$.

Пусть оператор $A \in$ End $\mathcal{X}$, и его матрица $\mathcal{A}$ является матрицей с суммируемыми диагоналями. Тогда оператор $A$ принадлежит пространству $\operatorname{End}_{1} \mathcal{X} \subset$ End $\mathcal{X}$ операторов, имеющих матрицы с суммируемыми диагоналями.

Лемма 7. Любая матрица $\mathcal{A} \in \operatorname{Matr}\left(\mathbb{J}^{2}, \mathbb{C}\right)$ с единственной ненулевой $k$-й диагональю $(k \in \mathbb{Z})$, элементы которой равномерно ограничены, определяет ограниченный оператор в любом из пространств $l_{p}(\mathbb{J}), 1 \leqslant p \leqslant \infty$, с нормой, не превосходлщей $d_{\mathcal{A}}(k)$.

Доказательство. Пусть матрица $\mathcal{A} \in \operatorname{Matr}\left(\mathbb{J}^{2}, \mathbb{C}\right)$ имеет ненулевую $k$-ю диагональ,

$$
\mathcal{A} x(n)=\mathcal{A}(k+n, n) x(n), \quad n \in \mathbb{J} ;\|A x\|_{p}=\left\|\sum_{k \in \mathbb{Z}} \mathcal{A}(k+n, n) x(n)\right\|_{p} \leqslant d_{\mathcal{A}}(k)\|x\|_{p},
$$

где $x \in l_{p}(\mathbb{J}), 1 \leqslant p \leqslant \infty$; отсюда и следует утверждение леммы.

Лемма 8. Матрица $\mathcal{A} \in \operatorname{Matr}\left(\mathbb{J}^{2}, \mathbb{C}\right)$ с суммируемыми диагоналями в любом из пространств $l_{p}, 1 \leqslant p \leqslant \infty$, является матрицей ограниченного оператора $A \in \operatorname{End} l_{p}(\mathbb{J})$ с нормой, удовлетворяющей оченке $\|A\| \leqslant\|A\|_{1}=d(\mathcal{A})$. 
Доказательство. Матрицу $\mathcal{A}$ представим в виде $\mathcal{A}=\sum_{k \in \mathbb{Z}} \mathcal{A}_{k}$, где каждая из матриц $\mathcal{A}_{k}$ имеет только одну ненулевую $k$-ю диагональ, совпадающую с $k$-й диагональю матрицы $\mathcal{A}$, а все остальные диагонали нулевые. Тогда из равенств

$$
\|A x\|_{p}=\left\|\sum_{k \in \mathbb{Z}} \mathcal{A}_{k} x\right\|_{p} \leqslant \sum_{k \in \mathbb{Z}} d_{\mathcal{A}}(k)\|x\|_{p}=d(\mathcal{A})\|x\|_{p}
$$

вытекает утверждение леммы.

Лемма 9. Спектр $\sigma(\mathcal{A})$ матрицы $\mathcal{A} \in \operatorname{Matr}\left(\mathbb{J}^{2}, \mathbb{C}\right)$ совпвдает со спектром $\sigma(A)$ оператора $A: D(A) \subset l_{p}(\mathbb{J}) \rightarrow l_{p}(\mathbb{J}), 1 \leqslant p \leqslant \infty$.

Приведем без доказательства используемый далее важный для нас результат (см. $[3,4])$.

Теорема 1 (см. [4, следствие 3]). Если оператор $A \in \operatorname{End}_{1} l_{q}(\mathbb{J})$ обратим при некотором $1 \leqslant q \leqslant \infty$, то он обратим во всех остальных пространствах $l_{p}(\mathbb{J}), 1 \leqslant p \leqslant \infty$.

Из теоремы 1 и леммы 9 вытекает следующее утверждение.

Лемма 10. Спектр $\sigma(\mathcal{A})$ матрицы $\mathcal{A} \in \operatorname{Matr}\left(\mathbb{J}^{2}, \mathbb{C}\right)$ не зависит от $p, 1 \leqslant p \leqslant \infty$.

2.2. Ряды Фуръе. Пусть $\mathcal{X}$ - абстрактное банахово пространство. Введем в рассмотрение периодическую периода $2 \pi$ группу $S(t), t \in \mathbb{R}$, действующую по формуле $(S(t) x)(k)=x(k) e^{i k t}, k \in \mathbb{J}$. Каждому ограниченному оператору $A \in \operatorname{End} \mathcal{X}$ поставим в соответствие функцию $\widetilde{A}: \mathbb{R} \rightarrow$ End $\mathcal{X}$, имеющую вид

$$
\Phi_{A}(t)=\widetilde{A}(t)=S(t) A S(-t), \quad t \in \mathbb{R} .
$$

Пусть функция $\widetilde{A}(t)$ имеет ряд Фурье вида

$$
\widetilde{A}(t) x \sim \sum_{k \in \mathbb{Z}} A_{k} x e^{i k t}, \quad x \in \mathcal{X}, \quad t \in \mathbb{R},
$$

где коэффициенты Фурье имеют вид

$$
A_{k}=\frac{1}{2 \pi} \int_{0}^{2 \pi} \widetilde{A}(t) x e^{-i k t} d t .
$$

Определение 16 (см. [3,4]). Ряд $\sum_{k \in \mathbb{Z}} A_{k}$ называется рядом Фурье оператора $A \in$ End $\mathcal{X}$, а операторы $A_{k} \in$ End $\mathcal{X}, k \in \mathbb{Z}$ - коэффициентами Фурье оператора $A$.

Следующая лемма аналогична [4, лемма 1].

Лемма 11. Каждый коэффиииент Фуръе $A_{k}, k \in \mathbb{Z}$ имеет матрицу $\mathcal{A}_{k}, k \in \mathbb{Z}$ с единственной ненулевой $k$-й диагональю, совпадающей с $k$-й диагональю матриць $\mathcal{A} \in \operatorname{Matr}\left(\mathbb{J}^{2}, \mathbb{C}\right)$ операmopa $A \in$ End $\mathcal{X}$.

Доказательство. Найдем элементы матрицы оператора $A_{k}, k \in \mathbb{Z}$. Согласно (2), (3)

$$
\begin{gathered}
\mathcal{A}_{k}(l, j)=\left(A e_{j}\right)(l), \quad l, j \in \mathbb{J}, \quad \mathcal{A}_{k}(l, j)=\frac{1}{2 \pi} \int_{0}^{2 \pi}\left(S(t) A S(-t) e_{j}\right)(l) e^{-i k t} d t, \\
\left(S(-t) e_{j}\right)=\sum_{m \in \mathbb{J}} e_{j}(m) e^{-i m t}=e_{j} e^{-i j t}, \quad A e_{j} e^{i j t}=\sum_{m \in \mathbb{J}} \mathcal{A}(m, j) e_{m} e^{-i j t} \\
\left(S(t) A e_{j} e^{i j t}\right)(l)=\mathcal{A}(l, j) e^{i l t} e^{-i j t} .
\end{gathered}
$$

Следовательно,

$$
\mathcal{A}_{k}(l, j)=\frac{1}{2 \pi} \int_{0}^{2 \pi} \mathcal{A} e^{i l t} e^{-i j t} e^{-i k t} d t= \begin{cases}\mathcal{A}(l, j) & \text { при } l-j=k \\ 0 & \text { в противном случае. }\end{cases}
$$


Важно отметить, что принадлежность матрицы оператора алгебре $\operatorname{End}_{1} \mathcal{X}$ означает суммируемость коэффициентов Фурье этого оператора. Важным является тот факт, что $\operatorname{End}_{1} \mathcal{X}$ является банаховой алгеброй. Действительно (см. [4]), пусть $A \in \operatorname{End}_{1} \mathcal{X}, B \in \operatorname{End}_{1} \mathcal{X}$,

$$
\Phi_{A}(t)=\sum_{k \in \mathbb{Z}} A_{k} e^{i k t}, \quad \Phi_{B}(t)=\sum_{k \in \mathbb{Z}} B_{k} e^{i k t},
$$

$C=B A$, то $C \in \operatorname{End} \mathcal{X}$. Тогда $\Phi_{C}=\Phi_{B} \Phi_{A}$. По теореме об умножении рядов Фурье векторных функций получаем, что функция $\Phi_{C}$ имеет абсолютно сходящийся ряд Фурье,

$$
\Phi_{C}(t)=\sum_{n \in \mathbb{Z}} C_{n} e^{i n t}, \quad t \in \mathbb{R}, \quad \text { где } \quad C_{n}=\sum_{j \in \mathbb{Z}} B_{n-j} A_{j} .
$$

Поэтому оператор $C \in \operatorname{End} \mathcal{X}$ имеет ряд Фурье вида

$$
C=\sum_{n \in \mathbb{Z}} C_{n}, \quad\left\|C_{n}\right\| \leqslant \sum_{j \in \mathbb{Z}}\left\|B_{n-j}\right\|\left\|A_{j}\right\|
$$

следовательно, $C \in \operatorname{End}_{1} \mathcal{X}$.

2.3. Метод подобных операторов. Начнем с определения подобных операторов.

Определение 17 (см. [2]). Два линейных оператора

$$
A_{1}: D\left(A_{1}\right) \subset \mathcal{X} \rightarrow \mathcal{X}, \quad A_{2}: D\left(A_{2}\right) \subset \mathcal{X} \rightarrow \mathcal{X}
$$

называются подобными, если существует такой непрерывно обратимый оператор $U \in \operatorname{End} \mathcal{X}$, что $U\left(D\left(A_{2}\right)\right)=D\left(A_{1}\right)$ и

$$
A_{1} U x=U A_{2} x, \quad x \in D\left(A_{2}\right) .
$$

Оператор $U$ называется оператором преобразования оператора $A_{1}$ в оператор $A_{2}$.

Обычно преобразованием подобия оператор $A_{1}$ приводят к оператору $A_{2}$, более просто устроенному. Например, оператор $A_{2}$ имеет матрицу диагонального или блочно-диагонального вида относительно некоторого базиса. У оператора $A_{2}$ в этом случае легко считаются спектральные характеристики. А так как спектры подобных операторов совпадают, то известен становится и спектр оператора $A_{1}$.

Соотношение (4) иногда называют сплетающим свойством (см. [13]), оператор преобразования $U$ называется также transmutation и говорят, что он сплетает операторы $A_{1}$ и $A_{2}$ (interwining operator). Историю и современное состояние теории операторов преобразования можно найти в [13]. Отметим, что операторы преобразования - более широкое понятие, чем то, которое дано в определении 17 , так как обратный к оператору в общем случае не обязан существовать и быть ограниченным.

Далее мы рассматриваем подобные операторы в смысле определения 17.

Пусть в абстрактном банаховом пространстве $\mathcal{X}$ рассматривается линейный замкнутый оператор $A: D(A) \subset \mathcal{X} \rightarrow \mathcal{X}$, имеющий спектр $\sigma(A)$ и непустое резольвентное множество $\rho(A)$. Оператор $A$ будем называть невозмущенным оператором. Возмутим оператор $A$ оператором $B$ из End $\mathcal{X}$ и будем рассматривать возмущенный оператор $A-B: D(A) \subset \mathcal{X} \rightarrow \mathcal{X}$.

Перейдем к формулировке основных определений метода подобных операторов, и в первую очередь определим пространство допустимых возмущений, а также операторы, осуществляющие преобразование подобия. Все это образует для невозмущенного оператора $A$ так называемую допустимую тройку.

Определение 18 (см. [2]). Пусть $\mathfrak{A} \subset$ End $\mathcal{X}$ - банахово пространство со своей нормой $\|\cdot\|_{*}$ и $J, \Gamma \in$ End $\mathfrak{A}$ - трансформаторы (т.е. линейные операторы, действующие в пространстве операторов). Тройку $(\mathfrak{A}, J, \Gamma)$ назовем допустимой тройкой для невозмущенного оператора $A: D(A) \subset$ $\mathcal{X} \rightarrow \mathcal{X}$, а $\mathfrak{A}$ - пространством допустимых возмущений, если выполнены следующие условия:

(i) $J$ и $\Gamma$ - непрерывные трансформаторы, причем $J$-проектор, $J^{2}=J$; 
(ii) $(\Gamma X) D(A) \subset D(A)$ и имеет место равенство

$$
A(\Gamma X)-(\Gamma X) A=X-J X
$$

для любого $X \in \mathfrak{A}$, выполняемое на векторах из $D(A)$, причем $Y=\Gamma X$-единственное решение уравнения $A Y-Y A=X-J X$, удовлетворяющее условию $J Y=0$;

(iii) $X(\Gamma Y),(\Gamma X) Y \in \mathfrak{A}$ для любых $X, Y \in \mathfrak{A}$, и существует такая постоянная $\gamma>0$, что $\|\Gamma\| \leqslant \gamma$ и $\max \left\{\|X \Gamma Y\|_{*},\|(\Gamma X) Y\|_{*}\right\} \leqslant \gamma\|X\|_{*}\|Y\|_{*}$ для любых $X, Y \in \mathfrak{A}$;

(iv) $J((\Gamma X) J Y)=0$ для всех $X, Y \in \mathfrak{A}$;

(v) для любого $X \in \mathfrak{A}$ и $\varepsilon>0$ можно указать такое число $\lambda_{\varepsilon} \in \rho(A)$, что $\left\|X\left(A-\lambda_{\varepsilon} I\right)^{-1}\right\|<\varepsilon$, где $I$ - тождественный оператор.

Отметим, что для одного невозмущенного оператора $A$ можно построить несколько разных допустимых троек.

Зафиксируем некоторую допустимую тройку $(\mathfrak{A}, J, \Gamma)$ для невозмущенного оператора $A$ и выведем основное уравнение метода.

Будем искать оператор преобразования оператора $A-B$ в более просто устроенный оператор $A-J X$ в виде $U=I+\Gamma X$, тогда с учетом пункта іі определения 18 имеем

$$
\begin{aligned}
& (A-B)(I+\Gamma X)-(I+\Gamma X)(A-J X)= \\
& =A-B+A \Gamma X-B \Gamma X-A-\Gamma X A+J X+\Gamma X J X=X-B-B \Gamma X+\Gamma X J X .
\end{aligned}
$$

Из этого следует, что операторы $A-B$ и $A-J X$ подобны и $U=I+\Gamma X-$ оператор преобразования, если $X \in \mathfrak{A}$ есть решение нелинейного операторного уравнения

$$
X=B \Gamma X-\Gamma X J X+B .
$$

\section{3. Основные результаты.}

3.1. Матричный и операторный подход в доказательстве теоремы Винера об абсолютно сходящихся рядах Фурье. Пусть $\mathcal{L}^{2}=\mathcal{L}^{2}[0,2 \pi]$ - гильбертово пространство измеримых по Лебегу на $[0,2 \pi]$ со значениями в $\mathbb{C}$ суммируемых с квадратом модуля (классов эквивалентности) функций. Скалярное произведение и порожденная им норма в $\mathcal{L}^{2}$ задаются соответственно формулами

$$
(x, y)=\frac{1}{2 \pi} \int_{0}^{2 \pi} x(t) \overline{y(t)} d t, \quad\|x\|_{2}^{2}=\frac{1}{2 \pi} \int_{0}^{2 \pi}|x(t)|^{2} d t, \quad x, y \in \mathcal{L}^{2} .
$$

Введенное пространство $\mathcal{L}^{2}$ изометрически изоморфно гильбертову пространству $\mathcal{L}_{2 \pi}^{2}(\mathbb{R}, \mathbb{C})$ периодических периода $2 \pi$, определенных на всей оси $\mathbb{R}$ со значениями в $\mathbb{C}$ комплексных функций, суммируемых с квадратом модуля на $[0,2 \pi]$.

В силу равенства Парсеваля пространство $\mathcal{L}_{2 \pi}^{2}(\mathbb{R}, \mathbb{C})$ изометрически изоморфно также пространству $l_{2}=l_{2}(\mathbb{Z})$. Изоморфизм задается формулой

$$
x \longmapsto\{\hat{x}(n), n \in \mathbb{Z}\}, \quad \hat{x}(n)=\frac{1}{2 \pi} \int_{0}^{2 \pi} x(t) e^{-i n t} d t ;
$$

здесь $\hat{x}(n)$ - коэффициенты Фурье функции $x \in \mathcal{L}_{2 \pi}^{2}(\mathbb{R}, \mathbb{C})$,

$$
x(t) \sim \sum_{n \in \mathbb{Z}} \hat{x}(n) e^{i n t} .
$$

Отметим, что последовательность функций $\left\{e^{i n t}, n \in \mathbb{Z}\right\}, t \in[0,2 \pi]$ является ортонормированным базисом в пространстве $\mathcal{L}^{2}$, а изоморфизм (6), опять же в силу равенства Парсеваля, переводит этот базис в стандартный базис $e_{j}, j \in \mathbb{Z}, e_{j}(i)=\delta_{i j}, i, j \in \mathbb{Z}, \delta_{i j}-$ символ Кронекера, пространства $l_{2}$. 
Символом $C_{2 \pi}(\mathbb{R}, \mathbb{C}) \subset \mathcal{L}_{2 \pi}^{2}(\mathbb{R}, \mathbb{C})$ обозначим банахово пространство непрерывных на $\mathbb{R}, 2 \pi$ периодических функций со значениями в $\mathbb{C}$ и нормой

$$
\|x\|_{\infty}=\max _{t \in \mathbb{R}}|x(t)|, x \in C_{2 \pi}(\mathbb{R}, \mathbb{C}) .
$$

Итак, задача состоит в следующем. Пусть функция $f$ из $C_{2 \pi}(\mathbb{R}, \mathbb{C})$ имеет ряд Фурье $f(t) \sim$ $\sum_{n \in \mathbb{Z}} c_{n}(f) e^{i n t}$, где $c_{n}(f), n \in \mathbb{Z}$, ее коэффициенты Фурье, причем $\sum_{n \in \mathbb{Z}}\left|c_{n}(f)\right|<\infty$. Пусть также $f$ не обращается в нуль на отрезке $[0,2 \pi]$. Обозначим $f^{-1}=1 / f$, необходимо оценить коэффициенты Фурье функции $f^{-1} \in C_{2 \pi}(\mathbb{R}, \mathbb{C})$.

Теорема 2 (теорема Винера; см. [14]). Пусть ряд Фуръе функиии $f \in C_{2 \pi}(\mathbb{R}, \mathbb{C})$ абсолютно сходится и $f(x) \neq 0$ для всех $x \in[0,2 \pi]$. Тогда ряд Фурье для функиии $f^{-1}$ также абсолютно сходится.

Доказательство. В гильбертовом пространстве $\mathcal{L}_{2 \pi}^{2}(\mathbb{R}, \mathbb{C})$ рассмотрим оператор $A_{f}$ умножения на функцию $f \in C_{2 \pi}(\mathbb{R}, \mathbb{C})$, действующий по формуле $\left(A_{f} x\right)(t)=f(t) x(t), t \in \mathbb{R}$.

Непосредственный подсчет показывает, что оператор $A$ имеет матрицу Лорана вида

$$
\left(\begin{array}{cccc}
c_{0}(f) & c_{-1}(f) & c_{-2}(f) & \ldots \\
c_{1}(f) & c_{0}(f) & c_{-1}(f) & \ldots \\
0 & c_{1}(f) & c_{0}(f) & \ldots \\
\vdots & \vdots & \ddots & \vdots
\end{array}\right)
$$

где $c_{i}(f), i \in \mathbb{Z}$ - коэффициенты Фурье функции $f \in C_{2 \pi}(\mathbb{R}, \mathbb{C})$. Иными словами, у матрицы $\mathcal{A}$ оператора $A$ элементы, стоящие на $k$-й диагонали, одинаковы и равны $k$-му коэффициенту Фурье функции $f$. Так как ряд Фурье функции $f$ абсолютно сходится, то матрица $\mathcal{A}$ является матрицей с суммируемыми диагоналями.

По условию функция $f$ не обращается в нуль, поэтому оператор $A_{f}$ обратим, обратным является оператор $A_{f^{-1}}$. Оператор $A_{f^{-1}}$ также имеет матрицу Лорана, у этой матрицы элементы, стоящие на $k$-й диагонали, одинаковы и также равны $k$-му коэффициенту Фурье функции $f^{-1}$. Тогда в силу [4, теорема 4] матрица оператора $A_{f^{-1}}$ является матрицей с суммируемыми диагоналями, а, следовательно, функция $f^{-1}$ имеет абсолютно сходящийся ряд Фурье.

3.2. Уравнение Фридрихса. В этом разделе нас будет интересовать случай, когда $J X=0$ для любого $X \in \mathfrak{A}$. Тогда уравнение (5) примет вид

$$
X=B \Gamma X+B .
$$

Уравнение (7) называется уравнением Фридрихса (см. [3]). Заметим, что ранее в методе подобных операторов, который широко используется профессором А. Г. Баскаковым и его учениками для исследования различных классов дифференциальных и разностных операторов (см. $[5,6,10,11,18])$ не применялась модификация метода подобных операторов, связанная с уравнением Фридрихса (7).

Сформулируем и докажем соответствующую теорему.

Теорема 3. Пусть выполнено условие

$$
2 \gamma\|B\|<1 .
$$

Тогда оператори $A-B$ и $A$ подобны, имеет место равенство

$$
(A-B)\left(I+\Gamma X_{*}\right)=\left(I+\Gamma X_{*}\right) A,
$$

где $X_{*} \in \mathfrak{A}$ - решение уравнения (7). Решение $X_{*}$ можно найти методом простых итераций, положив $X_{1}=B, \ldots$ Отображение $\Phi(X)=B \Gamma X+B, \Phi: \mathfrak{A} \rightarrow \mathfrak{A}$ является сжимающим в шаре

$$
\mathfrak{B}=\left\{X \in \mathfrak{A}:\|X-B\|_{*} \leqslant\|B\|_{*}\right\} .
$$


Доказательство. Вначале заметим, что $\|X\|=\|X-B+B\| \leqslant 2\|B\|$ для любого $X \in \mathfrak{A}$. Далее используется принцип сжимающих отображений и теорема Банаха о неподвижной точке для доказательства существования решения уравнения (7). Таким образом, надо показать, что $\Phi(\mathfrak{B}) \subset \mathfrak{B}$ и $\|\Phi(X)-\Phi(Y)\|_{*} \leqslant q\|X-Y\|_{*}$, где $q<1$ для всех $X, Y \in \mathfrak{B}$. Действительно, с учетом неравенства (8):

$$
\begin{gathered}
\|\Phi(X)-B\|_{*} \leqslant\|B \Gamma X\|_{*} \leqslant 2\|B\|_{*}^{2} \gamma \leqslant\|B\|_{*}, \\
\|\Phi(X)-\Phi(Y)\|_{*}=\|B \Gamma(X-Y)\|_{*} \leqslant\|B\|_{*} \gamma\|X-Y\|_{*} \leqslant \frac{1}{2}\|X-Y\|_{*} .
\end{gathered}
$$

Итак, решение уравнения (7) при выполнении условия (8) существует в шаре (10).

Для доказательства (9) необходимо показать, что оператор $I+\Gamma X_{*}$ непрерывно обратим. Так как $\left\|\Gamma X_{*}\right\| \leqslant \gamma\left\|X_{*}\right\|_{*} \leqslant 2 \gamma\|B\|_{*}$, то при выполнении условия (8) $\left\|\Gamma X_{*}\right\|<1$ и

$$
\left(I+\Gamma X_{*}\right)^{-1}=\sum_{n=0}^{\infty}(-1)^{n}\left(\Gamma X_{*}\right)^{n} .
$$

Для полноты доказательства теоремы 3 осталось установить равенство $\left(I+\Gamma X_{*}\right)(D(A))=D(A)$. Из свойства iі определения 18 вытекает включение $\left(I+\Gamma X_{*}\right)(D(A)) \subseteq D(A)$. Осталось показать, что $\left(I+\Gamma X_{*}\right)^{-1}(D(A)) \subseteq D(A)$. Опять же используя свойство іi имеем для $\lambda \in \rho(A)$

$$
\begin{aligned}
\Gamma X_{*}(A-\lambda I)^{-1} & =(A-\lambda I)^{-1}(A-\lambda I) \Gamma X_{*}(A-\lambda I)^{-1}= \\
& =(A-\lambda I)^{-1}\left(X_{*}+\Gamma X_{*} A-\lambda \Gamma X_{*}\right)(A-\lambda I)^{-1}= \\
& =(A-\lambda I)^{-1}\left(X_{*}(A-\lambda I)^{-1}+\Gamma X_{*}\right) .
\end{aligned}
$$

Так как $\left\|\Gamma X_{*}\right\|<1$, то, используя свойство iv, можно выбрать такое $\lambda \in \rho(A)$, что

$$
\left\|X_{*}(A-\lambda I)^{-1}+\Gamma X_{*}\right\|<1
$$

тогда

$$
\left(I+\Gamma X_{*}\right)^{-1}(A-\lambda I)^{-1}=(A-\lambda I)^{-1}\left(I+X_{*}(A-\lambda I)^{-1}+\Gamma X_{*}\right)^{-1},
$$

это и означает выполнение включения $\left(I+\Gamma X_{*}\right)^{-1}(D(A)) \subseteq D(A)$. Теорема 3 доказана.

Далее для полноты изложения приведем также стандартный вариант метода подобных операторов (см. [10]).

Вернемся к уравнению (7), заметим, что

$$
J X=J(B \Gamma X)+J B,
$$

т. к. $J(\Gamma X J X)=0, X, Y \in \mathfrak{A}$. Подставим (11) в (7), получим следующее нелинейное уравнение для нахождения неизвестного оператора $X_{*} \in \mathfrak{A}$ :

$$
X=B \Gamma X-\Gamma X J B-\Gamma X J(B \Gamma X)+B=\Phi_{1}(X) .
$$

Теорема 4 (см. [10]). Пусть (A, $J, \Gamma)$ - допустимая для оператора $A$ тройка и $B$-некоторый оператор из пространства возмущений $\mathfrak{A}$. Тогда, если

$$
4 \gamma\|J\|\|B\|_{*}<1,
$$

то оператор $A-B$ подобен оператору $A-J X_{*}$, где $X_{*} \in \mathfrak{A}$-решение уравнения (12), и оно может быть найдено методом простых итераций, если положить $X_{0}=0, X_{1}=B, \ldots$ Преобразование подобия оператора $A-B$ в оператор $A-J X_{*}$ осуществляет оператор $I+\Gamma X_{*} \in$ End $\mathcal{X}$. Отображение $\Phi_{1}: \mathfrak{A} \rightarrow \mathfrak{A}$ является сжимающим в шаре $\mathfrak{B}=\left\{X \in \mathfrak{A}:\|X-B\|_{*} \leqslant 3\|B\|_{*}\right\}$.

3.3. Диагонализация. В этом разделе рассматривается приведение к диагональному виду возмущенных диагональных матриц, где в качестве возмущения берутся строго нижнетреугольные матрицы с суммируемыми диагоналями.

Пусть теперь $\mathbb{J}=\mathbb{N}$, и все рассматриваемые далее в статье нижнетреугольные матрицы принадлежат пространству $\operatorname{Matr}\left(\mathbb{N}^{2}, \mathbb{C}\right)$.

Лемма 12. Каждая нижнетреугольная матрица $\mathcal{A} \in \operatorname{Matr}\left(\mathbb{N}^{2}, \mathbb{C}\right)$ с равномерно ограниченными элементами правильнал, если у нее нет нулевых строк. 
Доказательство. Пусть $\mathcal{A}$ - нижнетреугольная матрица, тогда

$$
(A x)(n)=\sum_{i=1}^{n} A(i, j) x(j), \quad x \in l_{p}, \quad n \in \mathbb{N} .
$$

Следовательно, ее строки суммируемы, и по лемме 6 она правильная.

Будем рассматривать операторы, построенные по этим матрицам.

Определение 19. Оператор, у которого матрица нижнетреугольная, называется каузальным или причинным.

Другое определение каузальных операторов можно посмотреть в [7]. Применение каузальных операторов рассматривалось в [19]. Отметим также работу [17], в которой к исследованию операторов с нижнетреугольными матрицами применялся метод Фридрихса. Подчеркнем. что в этой работе на возмущение не ставилось условие суммируемости диагоналей и только доказано подобие возмущенного оператора $A-B$ невозмущенному оператору $A$ при выполнении определенного набора условий.

Отметим еще интересный факт, касающийся строго нажнетреугольных матриц. При умножении двух строго нижнетреугольных матриц с ненулевыми первыми диагоналями получается матрица строго нижнетреугольная с нулевой первой диагональю и ненулевыми остальными диагоналями. Таким образом, с каждой следующей операцией умножения в матрице-результате ненулевые диагонали будут спускаться все ниже.

Так как спектр оператора из $\operatorname{End}_{1} l_{p}(\mathbb{N})$ не зависит от $p$ (см. лемму 10), то удобнее всего все выкладки проводить для случая $p=2$ (таким образом, в качестве $\mathcal{X}$ выступает гильбертово пространство $\left.H=l_{2}(\mathbb{N})\right)$.

Итак, пусть $A: D(A) \subset l_{2}(\mathbb{N}) \rightarrow l_{2}(\mathbb{N})$ - линейный замкнутый оператор с дискретным спектром, действующий по формуле

$$
(A x)(n)=\mathcal{A}(n, n) x(n), \quad n \in \mathbb{N}, \quad x \in l_{2}(\mathbb{N}),
$$

где $\mathcal{A}(n, n), n \in \mathbb{N}$-элементы диагональной (невозмущенной) матрицы $\mathcal{A} \in \operatorname{Matr}\left(\mathbb{N}^{2}, \mathbb{C}\right.$ ) и $\mathcal{A}(i, i) \neq \mathcal{A}(j, j)$ при $i \neq j$. Область определения $D(A)$ задается условием

$$
D(A)=\left\{x \in l_{2}(\mathbb{N}), \sum_{n \in \mathbb{N}}|\mathcal{A}(n, n) x(n)|^{2}<\infty\right\} .
$$

Определение 20 (см. [16]). Оператор $A: D(A) \subset \mathcal{H} \rightarrow \mathcal{H}$, где $\mathcal{H}$ - гильбертово пространство, называется нормальным, если $D\left(A^{*}\right)=D(A)$ и $\|A x\|=\left\|A^{*} x\right\|$ для всех $x \in D(A)$.

В рассматриваемом случае оператор $A$ является нормальным.

Возмущением является строго нижнетреугольная матрица $\mathcal{B}$ с равномерно ограниченными элементами $\mathcal{B}(i, j), i, j \in \mathbb{N}$, диагонали которой являются суммируемыми, т.е. $B \in \operatorname{End}_{1} l_{2}(\mathbb{N})$.

Тогда спектр оператора $A$ состоит из простых собственных значений

$$
\sigma(A)=\bigcup_{n \in \mathbb{N}} \sigma_{n}=\bigcup_{n \in \mathbb{N}}\{\mathcal{A}(n, n)\}, \quad \sigma_{n}=\left\{\lambda_{n}\right\}=\{\mathcal{A}(n, n)\} .
$$

Обозначим через $d$ величину

$$
d=\operatorname{dist}\left(\sigma(A) \backslash \sigma_{n}, \sigma_{n}\right)>0,
$$

очевидно, что $d>0$ для всех $n \in \mathbb{N}$. Положим $\mathfrak{A}=\operatorname{End}_{1}^{0} l_{2}(\mathbb{N})$, где

$$
\operatorname{End}_{1}^{0} l_{2}(\mathbb{N})=\left\{X \in \operatorname{End}_{1} l_{2}(\mathbb{N}): \mathfrak{X}(i, j)=0, i \leqslant j\right\} .
$$

Другими словами, в качестве пространства допустимых возмущений выступает пространство операторов, имеющих строго нижнетреугольную матрицу с суммируемыми диагоналями. Норма в $\operatorname{End}_{1}^{0} l_{2}(\mathbb{N})$ задается формулой

$$
\|B\|_{*}=\sum_{l \geqslant 1} d_{\mathcal{B}}(l)
$$

Отметим, что последовательность $d_{\mathcal{B}}: \mathbb{Z} \rightarrow \mathbb{R}$ имеет ненулевые элементы только при $l \in \mathbb{N}$. 



го матрицу $\mathfrak{X}=(\mathfrak{X}(i, j)), i, j \in \mathbb{N}$, определим элементы матрицы оператора $\Gamma X$ формулой

$$
\Gamma X(i, j)=\frac{\mathfrak{X}(i, j)}{\mathcal{A}(i, i)-\mathcal{A}(j, j)}, \quad i>j, \quad i, j \in \mathbb{N} .
$$

Элементы $\Gamma X(i, j)$ определены корректно ввиду формулы (14). Пусть также $J X=0$ для всех $X \in \operatorname{End}_{1}^{0} l_{2} \mathbb{N}$. Оператор $\Gamma X$ также имеет строго нижнетреугольную матрицу с суммируемыми диагоналями, и имеет место оценка $\|\Gamma X\|_{*}=d^{-1}\|X\|_{*}$.

Теорема 5. Тройка $\left(\operatorname{End}_{1}^{0} l_{2} \mathbb{N}, J, \Gamma\right)$ является допустимой тройкой для оператора $A$, имеющего диагональную матричу, для которой выполняется условие (14).

Доказательство. Для доказательства теоремы осталось доказать выполнение условия v из определения допустимой тройки и включение $(\Gamma X)(D(A)) \subset D(A)$. Условие $\mathrm{v}$ выполняется ввиду того, что величина $\|X\|$ конечна, а величину $\left\|\left(A-\lambda_{\epsilon} I\right)^{-1}\right\|$ можно сделать малой за счет подходящего выбора числа $\lambda_{\epsilon} \in \rho(A)$. Например, если $A$ - самосопряженный оператор, то в качестве $\lambda_{\epsilon}$ можно брать число $\lambda_{\epsilon}=i N$, где $N \in \mathbb{N}$ - достаточно большое.

Представим оператор $\Gamma X(A-\lambda I)^{-1}$ в виде

$$
\begin{aligned}
\Gamma X(A-\lambda I)^{-1} & =(A-\lambda I)^{-1}(A-\lambda I) \Gamma X(A-\lambda I)^{-1}= \\
& =(A-\lambda I)^{-1}(A \Gamma X-\lambda \Gamma X)(A-\lambda I)^{-1}= \\
& =(A-\lambda I)^{-1}(X-J X+\Gamma X A-\lambda \Gamma X)(A-\lambda I)^{-1}= \\
& =(A-\lambda I)^{-1}\left((X-J X)(A-\lambda I)^{-1}+\Gamma X\right) .
\end{aligned}
$$

Отсюда немедленно следует, что $y=(A-\lambda I)^{-1} x \in D(A)$ и $\Gamma X(A-\lambda I)^{-1} x \in D(A)$ для $x \in l_{2} \mathbb{N}$. Теорема доказана.

Из теоремы 3 и теоремы 5 вытекает следующее утверждение.

Теорема 6. Пусть выполнено условие

$$
\|B\|_{*}<2 d
$$

Тогда оператор $A-B$ подобен невозмущенному оператору $A$.

Следствие 1. При выполнении условия (16) собственные значения операторов $A-B$ и $A$, определяемых матрицами $\mathcal{A}-\mathcal{B}$ и $\mathcal{A}$, совпадают.

Следствие 2. Собственные векторы $\widetilde{e}_{k}, k \in \mathbb{N}$ оператора $A-B$ задаются формулой

$$
\widetilde{e}_{k}=e_{k}+\Gamma X_{*} e_{k}, \quad k \in \mathbb{N},
$$

где $\left(e_{k}, k \in \mathbb{N}\right)$ - векторы стандартного ортонормированного базиса в $l_{2}(\mathbb{N})$.

Из теоремы 6 и леммы 10 получаем следующую теорему.

Теорема 7. Спектр матрицы $\mathcal{A}$ при выполнении условия (16) в любом из пространств $l_{p}(\mathbb{N})$, $1 \leqslant p \leqslant \infty$, состоит из простых изолированных собственных значений $\mathcal{A}(n, n), n \in \mathbb{N}$.

Отметим, что условие строгой нижнетреугольности матрицы $\mathcal{B}$ не является ограничительным. Пусть $\mathcal{B}$ - нижнетреугольная матрица с ненулевой главной диагональю. Тогда отнесем главную диагональ к невозмущенному оператору $A$, т.е. оператор $A$ теперь действует по формуле

$$
(A x)(n)=\mathcal{A}(n, n) x(n)-\mathcal{B}(n, n) x(n), \quad n \in \mathbb{N} .
$$

Так как $\mathcal{B} \in \operatorname{End}_{1} l_{2}(\mathbb{N})$ и элементы матрицы $\mathcal{B}$ равномерно ограничены, то такая замена невозмущенного оператора корректна. В этом случае надо требовать выполнения условия (14) для нового, полученного невозмущенного оператора. 
3.4. Блочная диагонализация. В этом разделе рассматривается приведение оператора $A-B$ к блочно-диагональному виду. Пусть нормальный оператор $A$ имеет простые изолированные собственные значения $\lambda_{n}=\mathcal{A}(n, n), n \in \mathbb{N}$, но при этом выполняется более сильное условие

$$
\operatorname{dist}\left(\left\{\lambda_{n}\right\}, \sigma(A) \backslash\left\{\lambda_{n}\right\}\right) \rightarrow \infty, \quad n \rightarrow \infty,
$$

вместо условия (14). Условие (18) означает, что собственные значения оператора $A$ (диагональные элементы матрицы $\mathcal{A}$ ) «разбегаются». В этом случае для построения преобразования подобия оператора $A-B$ в оператор, имеющий матрицу блочно-диагонального вида, будем строить другую допустимую тройку с ненулевым трансформатором $J \in \operatorname{End}\left(\operatorname{End}_{1}^{0} l_{2}(\mathbb{N})\right)$ и потом использовать теорему 3. Уравнение Фридрихса в этом случае применяться не будет. Важно отметить, что оператор, к которому будем преобразованием подобия приводить оператор $A$, будет иметь матрицу, отличающуюся от матрицы $\mathcal{A}$ оператора $A$ на оператор конечного ранга. Условия на матрицу $\mathcal{B}$ оператора-возмущения $B$ остаются неизменными, а именно, $\mathcal{B} \in \operatorname{End}_{1}^{0} l_{2}(\mathbb{N})$.

Перейдем к построению допустимой тройки. Как и в прошлый раз, $\mathfrak{A}=\operatorname{End}_{1}^{0} l_{2}(\mathbb{N})$. Пусть $P_{n}=$ $P(\{\mathcal{A}(n, n)\}, A), n \in \mathbb{N}$ - спектральный проектор оператора $A$, построенный по спектральному множеству $\sigma_{n}=\{\mathcal{A}(n, n)\}, n \in \mathbb{N}$. Введем обозначение

$$
P_{(n)}=\sum_{i=1}^{n} P_{i}, \quad n \in \mathbb{N}
$$

Рассмотрим семейство трансформаторов $J_{n}, \Gamma_{n} \in \operatorname{End}_{1}^{0}\left(l_{2}(\mathbb{N})\right), n \geqslant 1$, действующих на оператор $X \in \operatorname{End}_{1}^{0}\left(l_{2}(\mathbb{N})\right.$ по формулам

$$
\begin{gathered}
J_{n} X=P_{(n)} X P_{(n)}+\sum_{i>n} P_{i} X P_{i} \\
\Gamma_{n} X=\Gamma X-\Gamma\left(P_{(n)} X P_{(n)}\right)=\Gamma\left(X-P_{(n)} X P_{(n)}\right), \quad n \in \mathbb{N}, \quad X \in \operatorname{End}_{1}^{0}\left(l_{2}(\mathbb{N})\right) .
\end{gathered}
$$

В предыдущем разделе показано, что $\Gamma X \in \operatorname{End}_{1}^{0}\left(l_{2}(\mathbb{N})\right.$ и $\|\Gamma\|=d^{-1}$, где $d$ определяется формулой (15).

Трансформаторы $J X$ и $Г X$ отличаются от трансформаторов $J_{n} X$ и $\Gamma_{n} X$ на оператор конечного ранга. Поэтому все свойства трансформатора $\Gamma_{n} X$, отмеченные ранее, остаются в силе. Трансформаторы семейства $J_{n} X$ имеют ненулевые элементы только в правом верхнем квадрате порядка $n$, а все остальные их матричные элементы равны нулю. Элементы матрицы трансформаторов $J_{n} X$ и $\Gamma_{n} X$ определяются формулами

$$
\begin{aligned}
& \left(J_{n} X\right)(i, j)= \begin{cases}\mathfrak{X}(i, j), & \max \{i, j\} \leqslant n, \\
0, & \text { в остальных случаях; }\end{cases} \\
& \left(\Gamma_{n} X\right)(i, j)= \begin{cases}\frac{\mathfrak{X}(i, j)}{\mathcal{A}(i, i)-\mathcal{A}(j, j)}, & \max \{i, j\}>n, i \neq j, \\
0, & \text { в остальных случаях. }\end{cases}
\end{aligned}
$$

Отсюда получаем оценку

$$
\left\|\Gamma_{n} X\right\|_{*} \leqslant|\mathcal{A}(n, n)-\mathcal{A}(n+1, n+1)|^{-1}\|X\|_{*} .
$$

Таким образом, величина $\gamma=\gamma_{n}$ из определения 18 равна $\gamma_{n}=|\mathcal{A}(n, n)-\mathcal{A}(n+1, n+1)|^{-1}, n \in \mathbb{N}$, и ее можно сделать малой при подходящем выборе числа $n \in \mathbb{N}$ за счет выполнения условия (18).

Следующее утверждение аналогично теореме 5.

Теорема 8. Тройка $\left(\operatorname{End}_{1}^{0}\left(l_{2}(\mathbb{N})\right), J_{n}, \Gamma_{n}\right)$ является допустимой тройкой для оператора $A$ при любом $n \in \mathbb{N}$.

Так как $J_{n} X \neq 0$ для $X \in \operatorname{End}_{1}^{0}\left(l_{2}(\mathbb{N})\right)$ и, в частности, $J_{n} B \neq 0$, то мы далее воспользуемся теоремой 3.

Из теорем 3 и 7 получаем следующий факт. 
Теорема 9. Существует такое натуральное число $n \in \mathbb{N}$, что оператор $A-B$ подобен блочно-диагональному оператору $A-J_{n} X_{*}$, где $X_{*} \in \operatorname{End}_{1}^{0}\left(l_{2}(\mathbb{N})\right)$ есть решение нелинейного операторного уравнения (12) с трансформаторами $J_{n} u \Gamma_{n}$ такими, что матрицы операторов $J_{n} X$ $u \Gamma_{n} X$ определяются формулами (19) и (20). Имеет место равенство

$$
(A-B)\left(I+\Gamma_{n} X_{*}\right)=\left(I+\Gamma_{n} X_{*}\right)\left(A-J_{n} X_{*}\right) .
$$

Оператор $X_{*} \in \operatorname{End}_{1}^{0}\left(l_{2}(\mathbb{N})\right)$ можно найти методом простых итераций, полагая $X_{0}=0, X_{1}=B$.

Отметим, что выполнение условия теоремы 3 , гарантирующего разрешимость нелинейного операторного уравнения (13), очевидно за счет малости нормы оператора $\Gamma_{n}$ - величины $\gamma_{n}$.

Теорема 10. Собственные значения $\widetilde{\lambda}_{k}, k \in \mathbb{N}$, оператора $A-B$ совпадают с собственными значениями $\lambda_{k}, k \in \mathbb{N}$, оператора $A$. Соответствующие собственные векторы $\widetilde{e}_{k}, k \in \mathbb{N}, n р и$ $k>n$ определяются формулой (17). Имеет место оценка

$$
\left\|\widetilde{e}_{k}-e_{k}-\Gamma_{n} B e_{k}\right\| \leqslant O\left(\gamma_{n}\right), \quad k>n .
$$

Для доказательства формулы (21) заметим, что из (17) следует равенство

$$
\widetilde{e}_{k}=e_{k}+\Gamma_{n} B e_{k}+\Gamma(X-B) e_{k},
$$

и последнее слагаемое имеет порядок $O\left(\gamma_{n}\right)$.

\section{СПИСОК ЛИТЕРАТУРЫ}

1. Баскаков А. Г. Методы абстрактного гармонического анализа в теории возмущений линейных операторов// Сиб. мат. ж. - 1983. - 24, № 1. - С. 21-39.

2. Баскаков А. Г. Гармонический анализ линейных операторов. - Воронеж, 1986.

3. Баскаков А. Г. Абстрактный гармонический анализ и асимптотические оценки элементов обратных матриц// Мат. заметки. - 1992. - 52, № 2. - С. 17-26.

4. Баскаков А. Г. Асимптотические оценки элементов матриц обратных операторов и гармонический анализ// Сиб. мат. ж. - 1997. - 38, № 1. - С. 14-28.

5. Баскаков А. Г., Ускова Н. Б. Обобщенный метод Фурье для системы дифференциальных уравнений первого порядка и группы операторов// Диффер. уравн. - 2018. - 54, № 2. - С. 276-280.

6. Баскаков А. Г., Ускова Н. Б. Спектральный анализ дифференциальных операторов с инволюцией и группы операторов // Диффер. уравн. - 2018. - 54, № 9. - С. 1287-1291.

7. Баскаков А. Г., Криштал И. А. Гармонический анализ каузальных операторов и их спектральные свойства// Изв. РАН. Сер. мат. - 2005. - 69, № 3. - С. 3-54.

8. Гантмахер Ф. Р. Теория матриц. - М.: Наука, 1967.

9. Гаркавенко Г. В. О диагонализации некоторых классов линейных операторов// Изв. вузов. Мат. 1994. - 11. - C. 14--19.

10. Гаркавенко Г. В., Ускова Н. Б. Метод подобных операторов в исследовании спектральных свойств разностного оператора с растущим потенциалом// Сиб. электрон. мат. известия. - 2017. - 14. C. $673-689$.

11. Гаркавенко Г. В., Ускова Н. Б. Спектральный анализ одного класса разностных операторов с растущим потенциалом// Изв. Саратов. ун-та. Нов. сер. Сер. Мат. Мех. Информ. - 2016. - 16, № 4 . C. $395-402$.

12. Мак-Кинси Дж. Введение в теорию игр. - М.: ГИФМЛ, 1960.

13. Катрахов В. В., Ситник C. М. Метод операторов преобразования и краевые задачи для сингулярных эллиптических уравнений// Совр. мат. Фундам. направл. - 2018. - 64, № 2. - С. 211-426.

14. Кахан Ж. П. Абсолютно сходящиеся ряды Фурье. - М.: Мир, 1976.

15. Кук Р. Бесконечные матрицы и пространства последовательностей. - М.: ГИФМЛ, 1960.

16. Рудин У. Функциональный анализ. - М.: Мир, 1975.

17. Скрынников $A$. В. О квазинильпотентном варианте метода Фридрихса в теории подобия линейных операторов// Функц. анал. прилож. - 1983. - 17, № 3. - С. 89--90.

18. Baskakov A. G., Krishtal I. A., Uskova N. B. Linear differential operator with an involution as a generator of an operator group// J. Oper. Matr. — 2018. — 12, № 3. - P. 723-756. 
19. Feintuch A., Saeks R. System theory. A Hilbert Space Approach. — New York-London: Academic Press, 1982.

20. Hinkkannen A. On the diagonalization of a certain class of operators// Michigan Math. J. - 1985. - 32, № 3. - P. 349-359.

Баскаков Анатолий Григорьевич

Воронежский государственный университет

E-mail: anatbaskakov@yandex.ru

Гаркавенко Галина Валериевна

Воронежский государственный педагогический университет

E-mail: g.garkavenko@mail.ru

Ускова Наталья Борисовна

Воронежский государственный технический университет

E-mail: nat-uskova@mail.ru 